\title{
[18] Determination of Superoxide Dismutase Activity by Purely Chemical System Based on NAD(P)H Oxidation
}

\author{
By Francesco Paoletti and Alessandra Mocali
}

Introduction

Most of the currently employed methods for the assay of superoxide dismutase (SOD) activity in tissue extracts are based on the ability of these enzymes to inhibit a superoxide-driven reaction. The extent to which the rate of reaction is reduced can be taken as an indirect measurement of enzyme activity. The generation of superoxide can be achieved by either enzymatic or nonenzymatic systems ${ }^{1}$; subsequently a suitable detection method, whether colorimetric, polarographic, or luminometric according to different approaches, ${ }^{2}$ must be devised. Essential requirements for any assay are sensitivity, reliability, and simplicity of the procedure so that it can be easily performed in the laboratory without the aid of expensive equipment and reagents.

The present assay satisfies all these needs and allows the determination of minute amounts of SODs, such as $2 \mathrm{ng}$, which are far below of the detection limit of most employed methods. ${ }^{3}$ Moreover, there is another important aspect worth mentioning: the usual detectors like nitro blue tetrazolium (NBT), cytochrome $c$, or other chromogenic substrates, which are reduced by superoxide, might also be electron acceptors for reducing agents known to occur in biological samples. This fact will explain the difficulties, often encountered with these assays, in attaining saturation levels and reproducible titration curves. Our method, on the contrary, relies on the oxidation of $\mathrm{NAD}(\mathrm{P}) \mathrm{H}$, and this makes the detection less prone to interferences by aspecific reduction from cellular components. Besides. since $\mathrm{NAD}(\mathrm{P}) \mathrm{H}$ is the detector, measurements of $\mathrm{SOD}$ activities can easily be accomplished spectrophotometrically as for the majority of other enzymes.

The method. originally developed in our laboratory, ${ }^{4}$ consists of a purely chemical reaction sequence which generates superoxide from mo-

'J. M. McCord, M. Crapo, and I. Fridovich, in "Superoxide and Superoxide Dismutases" (A. M. Michelson. J. M. McCord, and I. Fridovich, eds.), p. 11. Academic Press, New York, 1977.

${ }^{2}$ L. Flohe and F. Ötung, this series, Vol. 105, p. 93.

${ }^{3}$ W. F. Beyer and I. Fridovich, Anal. Biochem. 161, 559 (1987).

F. Paoletti, D. Aldinucci, A. Mocali, and A. Caparrini, Anal. Biochem. 154, 536 (1986). 
lecular oxygen in the presence of EDTA, manganese(II) chloride, and mercaptoethanol. NAD(P)H oxidation is linked to the availability of superoxide anions in the medium. As soon as SOD is added to the assay mixture, it brings about the inhibition of nucleotide oxidation. Therefore, at high concentrations of the enzyme the absorbance at $340 \mathrm{~nm}$ remains unchanged, while in the control (no SOD added) absorbance decrease; according to predictable kinetics.

Measurements are carried out at physiological $\mathrm{pH}$ and consist of a single spectrophotometric step by which SOD activities are reliably and sensitively determined in both crude and pure enzyme preparations. This procedure, involving stable and inexpensive reagents, appears suitable for application in biochemistry, plant physiology, and clinical chemistry.

\section{Procedures}

\section{Preparation of Samples}

SODs are soluble enzymes and can be extracted from tissues after disaggregation and homogenization in suitable media. For small amounts of material, like cultured cells, leukocytes collected from a few millileters of peripheral blood, or bioptic fragments, treatment with ultrasonics or freezing and thawing might be more appropriate than mechanical homogenization. Simple lysis in hypotonic solutions is adopted for red cells. Tissue solubilization by detergents could also be performed since those commonly used do not interfere directly with the assay. Besides, the interactions of detergents with extract components may affect either $\mathrm{NAD}(\mathrm{P}) \mathrm{H}$ oxidation rates or the stability of some SOD preparations (for further information, see Precautions). Whole tissue extracts should be freed from either subcellular organelles or debris. according to the need. by centrifugation to obtain a clear and homogeneous supernatant for analysis.

Prior to the assay, tissue extracts should always be dialyzed or desalted through a Sephadex G-25 column in order to remove low molecular weight compounds that might have nonenzymatic SOD-like activity. The dialysis buffe- or the medium used to develop gel filtration must be keot and used to replace the sample in the control cuvette (see Conditions ror Assay). Provided the controls are suitable, the assay seems to be unaffected by most of the buffers and media commonly employed for tissue extraction; namely, Tris- $\mathrm{HCl}$ and disodium phosphate buffer $(0.1 \mathrm{M}, \mathrm{pH}$ 7.4), sodium acetate-acetic acid buffer $(0.1 M, \mathrm{pH} 5-8)$, phosphate-buf-

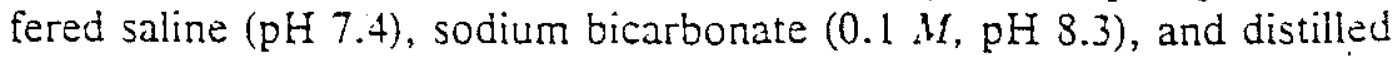


water have been individually used as sample solvents for several SOD preparations without any problem in activity determination, under the conditions given belôw.

Unless one is dealing with erythrocytes, specimens from laboratory animals should be removed by taking care to avoid trapped blood. Either perfusion or exhaustive wash of the tissue will prevent sample contamination by the red cell SOD and by excess hemoglobin. The latter interferes with the assay by counteracting the inhibitory effect of SOD; therefore, hemoglobin must be eliminated from the extract before SOD determinations. There are three different procedures, which can alternatively be used according to the need. The first two are useful for samples with a slight hemoglobin contamination (i.e., partially hemolysed plasmas, differentiated erythroleukemic cells, not well-perfused tissues).

The first approach is anion-exchange chromatography, using cellulose CM-52 (equilibrated with $8 \mathrm{~m} M$ Tris- $\mathrm{HCl}$ buffer, $\mathrm{pH}$ 6.8). Extracts to be treated must be first dialyzed or equilibrated with the developing buffer. The sample is applied to the column and eluted with Tris-HCl. Several proteins, including hemoglobin, bind to the cellulose while $\mathrm{Cu}, \mathrm{Zn}-\mathrm{SOD}$ and $\mathrm{Mn}-\mathrm{SOD}$ are completely recovered in the excluded fraction. This step is quite rapid and particularly suitable for small samples (up to $10 \mathrm{ml}$ ) which can be processed using a Pasteur pipet filled with approximately $0.5-1 \mathrm{~g}$ of wet cellulose.

The second method, gel filtration, takes advantage of differences in molecular weight among Mn-SOD $(\sim 86 \mathrm{~K})$, hemoglobin $(\sim 68 \mathrm{~K})$, and $\mathrm{Cu}, \mathrm{Zn}-\mathrm{SOD}(\sim 32 \mathrm{~K})$. To this purpose it is better to use a gel with a fractionation range of at least $5 \mathrm{~K}-150 \mathrm{~K}$, allowing a fine peak resolution. like Sephadex G-100 superfine or Sephacryl S-200.

The third procedure for hemoglobin removal, which applies to crude hemolysates and tissue extracts with high hemoglobin content, was originally developed by Tsuchihashi ${ }^{5}$ and further employed by McCord and Fridovich ${ }^{6}$ for the purification of $\mathrm{Cu}, \mathrm{Zn}$-SOD from erythrocytes. For clarity we report here an example of hemoglobin precipitation and SOD extraction from human erythrocytes (for values of SOD activity see Refeence Ranges). The hemolysate $(2.5 \mathrm{ml})$, prewarmed at $37^{\circ}$, is treated with $1 \mathrm{ml}$ of a mixture of ethanol-chloroform $(2: 1, \mathrm{v} / \mathrm{v})$ and mixed thoroughly to obtain a thick precipitate. Add $2 \mathrm{ml}$ of distilled water and mix again with the vortex. Incubate at $37^{\circ}$ for about $15 \mathrm{~min}$ with occasional stirring and then use a bench centrifuge to spin down the precipitate. The almost

\footnotetext{
${ }^{5}$ M. Tsuchihashi, Biochem. Z. 140, 65 (1963).

${ }^{6}$ J. M. McCord and I. Fridovich, J. Biol. Chem. 244, 6049 (1969).
} 
colorless supernatant is recovered, dialyzed, and then assayed after suitable dilution. However, because of the sensitivity of the method and high SOD levels in erythrocytes, human samples are usually diluted by such a large factor that dialysis might be omitted. The recovery of a standard of pure $\mathrm{Cu}, \mathrm{Zn}-\mathrm{SOD}$ added to the hemolysate is practically complete.

\section{Chemicals and Equipment}

Reduced adenine nucleotides ( $\beta-\mathrm{NADH}, \beta-\mathrm{NADPH}$, disodium salt), $\mathrm{MnCl}_{2} \cdot 4 \mathrm{H}_{2} \mathrm{O}$, 2-mercaptoethanol, ethylenediaminetetraacetic acid (EDTA), triethanolamine, and diethanolamine are all reagent grade and are used without further purification. Pure SOD preparations for standard calibration curves are prepared from bovine erythrocytes $\left(\sim 3,000\right.$ unit/mg lyophilizate, according to McCord and Fridovich ${ }^{6}$; Boehringer-Mannheim, West Germany). All solutions are made up with well-aerated distilled or deionized water. Measurements are performed with a spectrophotometer connected to a recorder and equipped with a multicell holder (at least 4 cells). A temperature control set unit may be useful.

\section{Reagents and Solutions}

Triethanolamine-diethanolamine (100 $\mathrm{m} M$ each)-HCl buffer (TDB): triethanolamine $(14.9 \mathrm{~g})$, diethanolamine $(10.5 \mathrm{~g})$, and approximately $13.8 \mathrm{ml}$ of concentrated $\mathrm{HCl}$ are dissolved in 1 liter of distilled water; the final $\mathrm{pH}$ should be around 7.4

$\mathrm{NAD}(\mathrm{P}) \mathrm{H}(7.5 \mathrm{mM})$ : dissolve $20 \mathrm{mg}$ of either $\mathrm{NADH}$ or NADPH, disodium salt (see Precautions), in $4 \mathrm{ml}$ of water (amount for 100 assays)

EDTA-MnCl $(100 \mathrm{~m} M / 50 \mathrm{mM})$ : prepare stock solutions of $200 \mathrm{mM}$ EDTA (dissolve $11.69 \mathrm{~g}$ EDTA-acid in $200 \mathrm{ml}$ water and adjust the $\mathrm{pH}$ to around 7 with $1 M \mathrm{NaOH}$ ) and $100 \mathrm{mM} \mathrm{MnCl}$ (dissolve 3.95 g $\mathrm{MnCl}_{2} \cdot 4 \mathrm{H}_{2} \mathrm{O}$ in $200 \mathrm{ml}$ water), combine the EDTA and $\mathrm{MnCl}_{2}$ stock solutions in a ratio of $1: 1(\mathrm{v} / \mathrm{v})$, and adjust the $\mathrm{pH}$ of the mixture (Reagent 3) to $\mathrm{pH} 7$ by dropwise addition of $10 \mathrm{M} \mathrm{NaOH}$ $(\sim 0.14 \mathrm{ml}$ per $25 \mathrm{ml}$ of reagent)

Mercaptoethanol $(10 \mathrm{~m} M): \mathrm{r}^{\prime \prime}$ ute $50 \mu$ l of concentrated thiol $(14.2 \mathrm{M})$ with $71 \mathrm{ml}$ of water

Stability of Solutions. TDB, mercaptoethanol, and both EDTA and $\mathrm{MnCl}_{2}$ stock solutions are quite stable even at room temperature if microbial contamination is avoided. Dissolved $\mathrm{NAD}(\mathrm{P}) \mathrm{H}$ can be kept at $4^{\circ}$ and used within 1 week. For longer storage, keep the coenzyme solution at $-20^{\circ}$. The EDTA- $\mathrm{MnCl}_{2}$ solution, once made up, is maintained at room temperature and can be used for over 1 month. Solutions of EDTA- 
$\mathrm{MnCl}_{2}$ may present some crystals, but these have no influence on the assay.

$\because$

\section{Conditions for Assay}

Each set of asșays must include its own control. The control consists of a cuvette where the sample is replaced by an equal volume of the medium used for enzyme solutions. The following solutions are subsequently pipetted into the cuvette (semimicrocuvette; light path $10 \mathrm{~mm}$ ): $0.8 \mathrm{ml} \mathrm{TDB}, 40 \mu \mathrm{l} \mathrm{NAD}(\mathrm{P}) \mathrm{H}, 25 \mu \mathrm{l}$ EDTA- $\mathrm{MnCl}_{2}$, and $0.1 \mathrm{ml}$ sample (or sample solvent for the control). Mix thoroughly and read at $340 \mathrm{~nm}$ against air for a stable baseline recorded over a 5 -min period. Within this interval the cuvettes will equilibrate at the right temperature (if needed), and the $\mathrm{NAD}(\mathrm{P}) \mathrm{H}$ oxidase activity possibly occurring in samples can be evaluated. Then add $0.1 \mathrm{ml}$ mercaptoethanol. The final volume in the cuvette is $1.065 \mathrm{ml}$. Mix and monitor the decrease in absorbance for about 20 min to allow full expression of the chain length leading to $\mathrm{NAD}(\mathrm{P}) \mathrm{H}$ oxidation.

A typical assay is reported in Fig. 1. Measurements of the relative

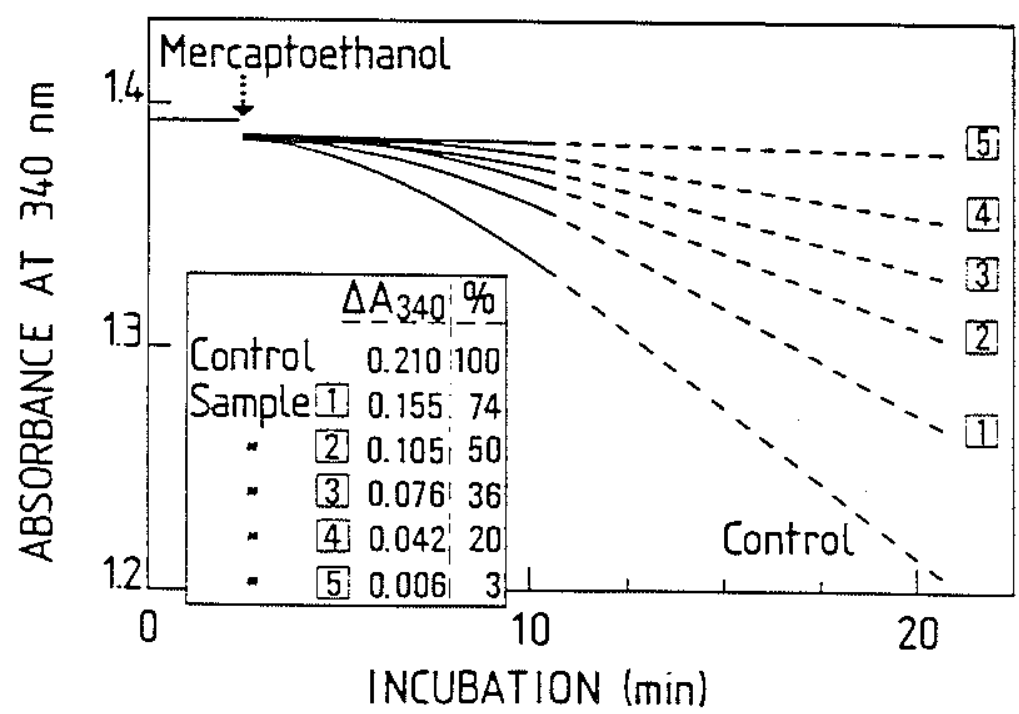

FIG. 1. Kineti - of NADH oxidation at different concentrations of SOD. Rates of NAJ:? oxidation reporteu concern six assays carried out simultaneously in the absence (Control) and in the presence of increasing amounts of pure $\mathrm{Cu}, \mathrm{Zn}-\mathrm{SOD}$ (sample 1, $12.5 \mathrm{ng}$; sample?. $31 \mathrm{ng}$; sample 3, $53 \mathrm{ng}$; sample 4, $95 \mathrm{ng}$; sample 5, $300 \mathrm{ng}$ of lyophilized enzyme, BoehringerMannheim). The reaction was started by the addition of mercaptoethanol. and the decrease in absorbance at $340 \mathrm{~nm}$ was recorded over $20 \mathrm{~min}$ at room temperature. The linear portions tion, curves used for calculation are indicated by dashed lines. The rates of NADH oxidaof inhibitin an interval of $8 \mathrm{~min}\left(\Delta A_{340}\right)$, are reported in the inset. together with percentages of inhibition relative to the control value. 


\section{Calculation}

One unit of SOD activity is defined as the amount of enzyme required to inhibit the rate of NAD(P)H oxidation of the control by $50 \%$. However, since calibration curves for all SOD preparations assayed attain saturation levels, the catalytic activity of the enzyme in the system will be equally expressed by either $50 \%$ or half-maximal inhibition. This means that the measurement of SOD in a preparation does not require running a full titration curve each time; an estimate of enzyme activity will be easily obtained by means of a single set of assays where serial sample dilutions (at least three) are challenged to reach half-maximal inhibition. Besides, for routine determinations on the same enzyme preparation, it might be convenient to have a calibration curve as a reference. This will allow extrapolation of $50 \%$ inhibition values in samples very low in enzyme activity. For practical purposes we have presented the data from the calibration curve of Fig. 2 in Table I, where percentages of inhibition and corresponding units of enzyme are reported together. The conversion

TABLE I

Conversion of Percentages of Inhibition to Linits of Enzymea

\begin{tabular}{ll|ll|ll|ll}
\hline$\%$ & Units & $\%$ & Units & $\%$ & Units & $\%$ & Units \\
\hline 96 & 0.064 & 80 & 0.323 & 64 & 0.590 & 48 & 1.078 \\
95 & 0.080 & 79 & 0.335 & 63 & 0.613 & 47 & 1.119 \\
94 & 0.096 & 78 & 0.348 & 62 & 0.636 & 46 & 1.162 \\
93 & 0.112 & 77 & 0.362 & 61 & 0.661 & 45 & 1.207 \\
92 & 0.128 & 76 & 0.375 & 60 & 0.686 & 44 & 1.253 \\
91 & 0.144 & 75 & 0.390 & 59 & 0.712 & 43 & 1.301 \\
90 & 0.160 & 74 & 0.405 & 58 & 0.740 & 42 & 1.350 \\
89 & 0.176 & 73 & 0.421 & 57 & 0.768 & 41 & 1.403 \\
88 & 0.192 & 72 & 0.437 & 56 & 0.797 & 40 & 1.457 \\
87 & 0.208 & 71 & 0.453 & 55 & 0.828 & 39 & 1.513 \\
86 & 0.225 & 70 & 0.471 & 54 & 0.860 & 38 & 1.571 \\
85 & 0.241 & 69 & 0.489 & 53 & 0.893 & 37 & 1.631 \\
84 & 0.257 & 68 & 0.508 & 52 & 0.927 & 36 & 1.694 \\
83 & 0.273 & 67 & 0.527 & 51 & 0.963 & 35 & 1.759 \\
82 & 0.289 & 66 & 0.547 & 50 & 1.000 & & \\
81 & 0.305 & 65 & 0.568 & 49 & 1.038 & & \\
\hline
\end{tabular}

${ }^{a}$ Conversion of percentages of inhibition to units of SOD activity was obtained by solving for $x$ in the following equations: $x=(99.974-y) / 2.003$, in the range 96-81 $x$ : and $\log (x)=(1+1.2649-y) / 61.156$, in the range $80-35.5$ : where $y$ is percent inhibition and $x$ is SOD concentration (ng). Units of SOD activity were calculated by dividing $x$ by $31 \mathrm{ng}$. which is the amount of pure enzyme yielding 50\% inhibition. namely, 1 unit of SOD. These results are also suitable for calculation of SOD activity, with good approximation. in rat liver extracts and in preparations of both $\mathrm{Cu}, \mathrm{Zn}$ - and $\mathrm{Mn}$-SOD from human leukocytes. 
rates in both the control and sample cuvettes are usually made on the straight portion of the curve, that is, starting from 5-10 min after the addition of mercaptoethanol. The rate of nucleotide oxidation of the control, calculated over an 8-min interval $\left(\Delta A_{340}\right)$, should be in the range of $0.12-0.35$. Values of $\Delta A_{340}$ recorded for samples with SOD activity will progressively decrease depending on the amount of enzyme in the assay mixture (see Fig. 1, inset). For the calculation, use (sample rate/control rate) $\times 100=\%$ inhibition.

The results of a calibration curve with pure $\mathrm{Cu}, \mathrm{Zn}$-SOD from bovine erythrocytes are shown in Fig. 2. Increasing amounts of enzyme (from 0 to $350 \mathrm{ng}$; abscissa) induce a proportionate decrease in the rate of $\mathrm{NAD}(\mathrm{P}) \mathrm{H}$ oxidation expressed as percentages of the control value (ordinate). With this enzyme preparation, $50 \%$ and almost complete saturation levels (98\%) were obtained with about 31 and 350 ng lyophilized protein, respectively.

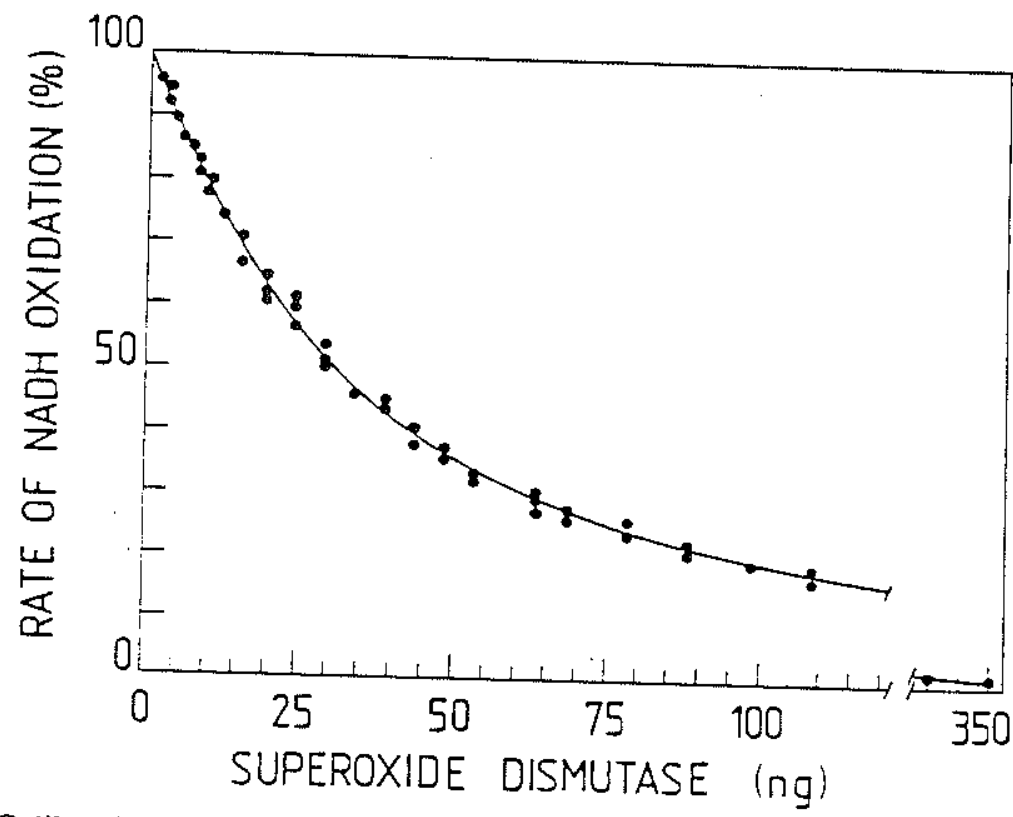

FIG. 2. Calibration curve of pure Cu.Zn-SOD. Increasing amounts $(0-350 \mathrm{ng})$ of pure enzyme from bovine erythrocytes (Boehringer-Mannheim) were assayed for activity under standard conditions as reported in the text, using NADH as the detector. The rates of $\mathrm{NADH}$ oxidation at $25^{\circ}$ were expressed as percentages of the control. Heat-inactivated SOD up to $350 \mathrm{ng}$ did not affect NADH oxidation at any rte (data not shown). The curve fitting experimental values in the range $10-55 \mathrm{ng}$ was obtained by least-squares regression analysis of SOD amounts transformed into logarithms: $y=141.2649-61.156 \log (x)$, where $y$ is percent inhibition and $x$ is nanograms of enzyme: the correlation coefficient $r$ is -0.9804 and $n$ is 29. The portion of the curve in the range $0-10 \mathrm{ng}$ is linear and represented by the equation $y=99.974-2.003 x$, where $r$ is -0.999 and $n$ is 10 . The last part of the curve. in the range $55-350 \mathrm{ng}$. has been traced by hand. With this enzyme preparation. $50 \%$ inhibition of NADH oxidation relative to the control was produced by approximately $31 \mathrm{ng}$ of lyophilized protein. Almost complete saturation levels (98\%) were obtained with $350 \mathrm{ng}$. 
table turns out to be quite useful for quick and accurate calculation of activity; especially in those portions of calibration curve where percentages of inhibition below $50 \%$ are difficult to assess.

Proper calculation of enzyme activity should take into account aspecific nucleotide oxidation possibly occurring in crude extracts. The contribution of $\mathrm{NAD}(\mathrm{P}) \mathrm{H}$ oxidase to the final rate must be evaluated before mercaptoethanol addition and eventually be subtracted. This precaution is unnecessary when assaying for clear diluted cytosols and pure SOD preparations.

Calibration curves obtained with several pure and crude SOD preparations are practically overlapping. Variations in the slope of the standard curve may result from changes in assay conditions. For a correct estimate of SOD activity in a sample it would be worth referring to a new calibration curve where assay conditions (temperature, sample buffer and volume, reagents, and type of extract) reflect those of routine determinations.

\section{Precautions and Special Conditions for Measurement}

Samples to be analyzed should not contain chelators which would alter EDTA $/ \mathrm{Mn}^{2+}$ ratios required for optimal nucleotide oxidation. Similarly, the presence of endogenous $\mathrm{Mn}^{2+}$ will affect the rate of the reaction. Other divalent cations of the second transition series do not interfere directly with $\mathrm{NAD}(\mathrm{P}) \mathrm{H}$ oxidation but could compete with $\mathrm{Mn}^{2-}$ for the chelator. Nucleotide oxidation rates are dependent on mercaptoethanol concentration in the assay mixture; therefore. samples should be devoid of free thiols which will increase oxidation rates over control values. To avoid all these problems and those derived from low molecular weight compounds with SOD-like activity, the sample should be dialyzed or desalted before the assay is performed. Several buffers and media (see Preparation of Samples) have been reliably employed as sample solvents.

The concentrations of urea and ethanol ( 0.6 and $1 M$, respectively) in the cuvette are compatible with reliable enzyme measurements. Detergents such as Triton X-100, Brij 35, sodium deoxycholate. Nonidet. digitonin. Tween 20. and SDS. at a final concentration of $0.1 \%$. can be included in the assay mixture without appreciable interferences in the SOD activity determination. However, Triton X-100 has been reported to catalyze NADH oxidation by artificial electron acceptors, ${ }^{7}$ and SDS was found to enhance membrane-bound NADPH oxidase activity in cell-free systems. ${ }^{8}$ In addition. SDS is also known to inactivate some Mn-SOD

7 U. M. Rao, Biocilem. Int. 5.585 (1982).

${ }^{8}$ Y. Bromberg and E. Pick. J. Biol. Chem. 260, 13539 (1985). 
preparations. ${ }^{9}$ Therefore, reagents used for either tissue solubilization or enzyme extraction, even without interfering directly with the assay, should be checked in advance to avoid misinterpretation of results.

Both NADH and NADPH are equally susceptible to oxidation in our system. There are crude extracts, however, which contain microsomal or plasma membrane fractions and thus show high levels of NADH or NADPH oxidase activities. In this case it may be worthwhile to run the assay with the nucleotide that is not the favored substrate for sampleoxidase to ensure low rates of aspecific oxidation.

Freshly made solutions of EDTA- $\mathrm{MnCl}_{2}$ show an initial delay in coenzyme oxidation; therefore, as pointed out above, linear kinetics are obtained only 5-10 min after mercaptoethanol addition. Conversely, aged preparations of EDTA- $\mathrm{MnCl}_{2}$ yield almost straight kinetics right from the beginning, thus reducing the time required for the assay. Slight variations in control maximal rates $\left(\Delta A_{340} \times 8 \mathrm{~min}\right)$ observed along with storage of the EDTA- $\mathrm{MnCl}_{2}$ reagent are without effect on the calculation of enzyme activity.

The presence of cyanide up to $0.2 \mathrm{mM}$ in the assay mixture is still compatible with the measurement even if it partially slows down the control rate. Conversely, concentrations of cyanide over $1 \mathrm{~m} M$ yield almost complete inhibition of nucleotide oxidation and cannot be used in the system. A protocol for differential measurements of SOD activity by cyanide inhibition experiments is described in the following paragraph.

Despite the sensitivity of the method, samples very low in SOD activity (e.g., the levels of both $\mathrm{Mn}$ - and $\mathrm{Cu} . \mathrm{Zn}$-SOD are greatly reduced in tumor tissue ${ }^{10}$ ) are difficult to assay. To overcome this problem the sample volume in the assay mixture could appropriately be increased up to 0.8 $\mathrm{ml}$ while the buffer (TDB) volume is reduced to $0.1 \mathrm{ml}$. Naturally, the sample medium should be such as to not substantially affect the final $\mathrm{pH}$ in the assay mixture. Whenever samples are diluted in $0.1 M$ TDB, the TDB in the assay can be omitted, and the procedure is carried out directly on $0.9 \mathrm{ml}$ of sample. The above approach can also be very useful in the determination of enzyme activity in chromatographic fractions when strikingly different levels of SOD isoenzymes are encountered within the ame eluate. We have been able to detect and estir te amounts of Mn-SOD as small as $0.1-0.4 \mathrm{U} / \mathrm{ml}$, separated from cell extracts where approximately $97 \%$ of the total SOD activity was due to the Cu.Zn isoenzyme.

${ }^{9}$ B. L. Geller and D. R. Winge. Anal. Biochem. 128, 86 (1983).

${ }^{10}$ L. W. Oberley, in "Superoxide Dismutase" (L. W. Oberley, ed.), Vol. 2. p. 127. CRC Press. Boca Raton. Florida, 1982. 
Enzyme Properties Relevant in Analysis

SODs in fresh tissue extracts are fairly stable, but they loose activity during storage and purification. The Mn-SOD isoenzyme, in particular, shows a varied degree of stability depending on the source from which it is extracted. Rat liver enzyme, for instance, is much more labile than human Mn-SOD. " A treatment with organic solvents as suggested for hemoglobin precipitation (ethanol-chloroform, 2:1), inactivates Mn-SOD and extracellular (EC) SOD, ${ }^{12}$ whereas $\mathrm{Cu}, \mathrm{Zn}$-SOD is usually unaffected. This is not a problem when assaying for activity in erythrocytes which contain only the $\mathrm{Cu}, \mathrm{Zn}$ isoenzyme, but it might be limiting for other cell types. The inactivation of Mn-SOD by SDS has been reported, ${ }^{9}$ but in our experience this effect cannot be taken as a general rule for discriminating between $\mathrm{Mn}$ - and $\mathrm{Cu}, \mathrm{Zn}-\mathrm{SOD}$ in any extract.

Cyanide is a powerful inhibitor of copper-containing SODs, and this is the basic principle of a widely used procedure for the discrimination between cyanide-sensitive ( $\mathrm{Cu}, \mathrm{Zn}$ and extracellular isoenzyme) and cyanide-insensitive ( $\mathrm{Mn}$ and $\mathrm{Fe}$ isoenzyme) SOD activity in tissue extracts. However, despite the selectivity of the cyanide effect, inhibition experiments do not always provide clear-cut results, owing to the relative amounts of isoenzymes present, the concentration of the inhibitor, and the time of incubation with the extract. There are also differences in both the sensitivity and detection limit as well as in the detection system of several methods available, making it difficult to evaluate the relative contribution of cyanide-sensitive and -insensitive SOD activity in a given sample. We describe herein a procedure which applies to the present method and has proved to be useful for isoenzyme discrimination. The typical experiment requires the incubation of an extract (up to 800 units $\mathrm{SOD} / \mathrm{ml}$ ) at $37^{\circ}$ with and without $1 \mathrm{~m} M \mathrm{NaCN}$ in $0.1 M$ TDB using stoppered test tubes. After $2 \mathrm{hr}$ samples are assayed for SOD activity, and relative rates of nucleotide oxidation are referred to those of controls containing the same amount of incubation buffers with and without cyanide. The difference in activities between samples with and without the inhibitor corresponds to that arising from the copper-containing SOD. inactivated by the treatment. Inh tion by cyanide of endogenous $\mathrm{Cu}, \mathrm{Zn}$ SOD in a rat liver extract and of pure $\mathrm{Cu}, \mathrm{Zn}$-SOD added to it as internal standard is over $97 \%$. On the contrary, Mn-SOD activity, estimated following chromatographic separation of the extract, is practically unaffected by cyanide.

"F. Paoletti and A. Mocali, unpublished observations (1988).

12 S. L. Marklund, Biochem. J. 220, 269 (1984). 


\section{Comments}

The precision of the method has been tested by assaying for $0.5,1$, and 1.5 units of pure $\mathrm{Cu}, \mathrm{Zn}-\mathrm{SOD}$, yielding a relative standard deviation (RSD) of $1.5,2$, and $2.5 \%$, respectively. The detection limit, $\Delta A_{340}$ per $8 \mathrm{~min}$, is about 0.01 , corresponding to approximately $2 \mathrm{ng}$ of standard enzyme (Boehringer preparation), and the sensitivity at $A_{3+1}$ is about 0.005 .

Inhibitors of the reaction are $\mathrm{Mn}^{2+}$, uric acid, ascorbic acid, hydrogen peroxide at millimolar levels, other possible superoxide scavengers, and low molecular weight compounds exhibiting SOD-like activity. Since $\mathrm{NAD}(\mathrm{P}) \mathrm{H}$ oxidation is oxygen dependent, the use of deaerated solutions also impairs the efficiency of the assay. Catalase does not interfere with the assay; in fact, it may be included in the mixture to lower hydrogen peroxide levels in the system.

Acid EDTA-Mn solutions increase their activity with time, whereas neutralized preparations, as reported here, yield more stable and constant kinetics during storage. The EDTA- $\mathrm{MnCl}_{2}$ reagent is the real trigger of all the reaction sequences; therefore, variations in EDTA-Mn solutions may lead to differences in NADH oxidation rates. However, for rates within a relatively wide range (see Conditions for Assay) there are no significant
changes in the SOD calibration curve.

Most of the inconsistencies in measurement depend on poor dialysis or desalting of samples and on the presence of hemoglobin, as already discussed. The fact that titrations of both crude and pure SOD preparations yield almost overlapping curves confirms the good specificity of the assay and the lack of gross interferences from physiological compounds occurring in tissue extracts. Moreover, the possibility of changing the standard assay conditions and measuring very low SOD activities on a time scale of minutes points out the flexibility of the method. It is also worth recalling that the physiological $\mathrm{pH}$ at which the assay is carried out allows a correct estimate of Mn-SOD activity that is not well-expressed in alkaline media as required by other sensitive methods. ${ }^{13.14}$

The assay is based on a purely chemical reaction sequence. and this excludes any possibility of introducing contaminating SOD activity in the system $y$ the use of impure preparations of auxiliary enzymes i cytochrome $c$. In addition, the sample assayed can be recovered from the cuvette and collected for further analysis after simple dialysis. Both these facts represent clear advantages of the chemical over enzymatic
methods.

is S. L. Marklund, J. Biol. Chem. 251. 7504 (1976).

${ }^{i t}$ H. P. Misra and I. Fridovich. J. Biol. Chem. 247, 3170 (1972). 


\title{
References Ranges
}

The specific activities of total SOD from various sources, according to the present method, are tabulated below. These values are intended only to provide references for practical purposes, and a certain degree of variability may be expected depending on the material and treatments employed.

\begin{tabular}{lcc}
\hline \multicolumn{1}{c}{ Source } & Activity \\
\hline Human plasma & $0.33 \mathrm{U} / \mathrm{mg}$ protein \\
Human erythrocytes & $11.5 \mathrm{U} / \mathrm{mg} \mathrm{Hb}$ \\
Human erythrocytes & $0.36 \mathrm{U} / 10^{6}$ cells \\
Human mononuclear leukocytes & 20 & $\mathrm{U} / \mathrm{mg}$ cytosolic protein \\
Rat liver (perfused) & 324 & $\mathrm{U} / \mathrm{mg}$ cytosolic protein \\
HL-60 (human promyelocytic cells) & 37 & $\mathrm{U} / \mathrm{mg}$ cytosolic protein \\
3T3-BALB (normal fibroblasts) & 63 & $\mathrm{U} / \mathrm{mg}$ cytosolic protein \\
3T3-B77 (transformed fibroblasts) & 27 & $\mathrm{U} / \mathrm{mg}$ cytosolic protein \\
NIH-3T3 KiMSV (transformed fibroblasts) & 26 & $\mathrm{U} / \mathrm{mg}$ cytosolic protein \\
Friend erythroleukemia cells & 45 & $\mathrm{U} / \mathrm{mg}$ cytosolic protein \\
Cu,Zn-SOD (Boehringer; Cat. 837 113) & 32,180 & $\mathrm{U} / \mathrm{mg}$ lyophilizate \\
\hline
\end{tabular}

\section{Acknowledgements}

This work was supported by grants from the Ministero della Pubblica Istruzione (60 and $40 \%$ ) and from the Associazione Italiana per la Ricerca sul Cancro (A.I.R.C.).

\section{[19] Assays for Superoxide Dismutase Based on Autoxidation of Hematoxylin}

\author{
By Joseph P. MARTIN, JR.
}

Introduction

Superoxide dismutase (SOD) catalyzes the reaction

$$
\mathrm{O}_{2}^{-}+\mathrm{O}_{2}^{-}+2 \mathrm{H}^{+} \rightarrow \mathrm{H}_{2} \mathrm{O}_{2}+\mathrm{O}_{2}
$$

A variety of spectrophotometric assays for the enzyme have been described. One category of assays combines superoxide-generating sys- 